\title{
Modification of Nanostructured Fused Silica For Use As Superhydrophobic, IR-Transmissive, Anti-Reflective Surfaces
}

Darryl A. Boyd ${ }^{1 *}$, Jesse A. Frantz ${ }^{1}$, Shyam S. Bayya ${ }^{1}$, Lynda E. Busse ${ }^{1}$, Woohong Kim ${ }^{1}$, Ishwar Aggarwal ${ }^{2}$, Menelaos Poutous ${ }^{2}$, and Jasbinder S. Sanghera ${ }^{1}$

${ }^{1}$ Optical Sciences Division, Naval Research Laboratory, 4555 Overlook Ave SW, Washington, DC 20375

${ }^{2}$ University of North Carolina, Charlotte, NC 28223

*darryl.boyd@nrl.navy.mil -202-404-6140

\section{Abstract}

In order to mimic and enhance the properties of moth eye-like materials, nanopatterned fused silica was chemically modified to produce self-cleaning substrates that have anti-reflective and infrared transmissive properties. The characteristics of these substrates were evaluated before and after chemical modification. Furthermore, their properties were compared to fused silica that was devoid of surface features. The chemical modification imparted superhydrophobic character to the substrates, as demonstrated by the average water contact angles which exceeded $170^{\circ}$. Finally, optical analysis of the substrates revealed that the infrared transmission capabilities of the fused silica substrates (nanopatterned to have moth eye on one side) were superior to those of the regular fused silica substrates within the visible and near-infrared region of the light spectrum, with transmission values of $95 \%$ versus $92 \%$, respectively. The superior transmission properties of the fused silica moth eye were virtually unchanged following chemical modification.

\section{Introduction}

Nature has provided numerous examples of materials with surfaces having nanoscale features that serve a practical survival purpose for living species. The material that comprises a moth's eye is one such surface. Upon close examination, it has been revealed that the moth eye is comprised of an array of nanoscopic features that are responsible for anti-reflection (AR) character.[1,2] The moth eye transmits a large fraction of incident light, allowing the moth to see in dimly lit areas.[3] The AR property of moth eye also allows moths to go visually undetected from predators.[4] In order to mimic the AR quality of moth eye, periodic and randomly 
nanopatterned surfaces have been produced in an array of materials.[4-8] Finally, though there are some research reports in which substrates are modified with a surface coating to impart moth eye-like AR properties,[4-6] directly patterning an $\mathrm{AR}$ structure into substrates is a preferable alternative because coatings are subject to delamination under thermal cycling.[9]

When nanoscale features are patterned into the surface of a transparent substrate, the shape and dimensions of those features determine the light transmission as a function of wavelength. These features may be ordered or random as long as the features are sub-wavelength in scale. Fused silica is a common substrate material that transmits in the near-infrared (NIR) region of the light spectrum. Although it has been observed that some materials, such as arsenic trisulfide $\left(\mathrm{As}_{2} \mathrm{~S}_{3}\right)$ glass fibers that were "stamped" or imprinted with a moth eye pattern exhibited superhydrophobicity,[10] fused silica is typically hydrophilic and exhibits superhydrophilicity when imprinted with a moth eye pattern.[11] However, if the surface is chemically modified with compounds that contain hydrophobic end groups, the surface could then be made to be hydrophobic.[11-14] In general, surface modified, nanopatterned materials may be useful in numerous field applications such as visible and infrared optical lenses that function in environments where visibility is obstructed due to moisture. Herein we outline a method for the chemical modification of moth eye patterned fused silica for use as self-cleaning, visible and near IR-transmissive, and anti-reflective surface coatings.

\section{Experimental}

Materials: $1 \mathrm{H}, 1 \mathrm{H}, 2 \mathrm{H}, 2 \mathrm{H}-$ perfluorooctyl trichlorosilane (PFOTS) and n-hexanes were purchased from SigmaAldrich and used as received. UV grade fused-silica substrates were each patterned with random moth eye structures on only one side.

Surface Modification of Moth Eye Substrate: UV grade fused-silica substrates, which were randomly prepatterned on one side, were subjected to an $\mathrm{O}_{2(\mathrm{~g})}$ atmosphere for 10 minutes in a March Plasma Reactive Ion Etcher under a pressure of 320 mTorr, and at a power of 200 Watts. The substrates were then removed, immersed and wafted for 30 seconds in hexanes that contained $0.5 \%$ PFOTS. The substrates were immediately rinsed with hexanes, blown dry with $\mathrm{N}_{2(\mathrm{~g})}$ and placed in an oven to bake for 15 minutes at $120{ }^{\circ} \mathrm{C}$. Finally, the substrates were removed from the oven and allowed to cool. 
Spectroscopic Data Collection: UV-visible-NIR data was obtained both before and after surface modification using an Agilent Technologies Cary 7000 Universal Measurement Spectrophotometer in the range of $250-2300$ nm. Specifically, percent transmission and percent reflectance data were obtained.

Scanning Electron Micrograph Images: Scanning Electron Micrograph (SEM) images of the substrate surfaces were taken using a Carl Zeiss LEO Supra 55 scanning electron microscope. Images were taken at $0^{\circ}, 10^{\circ}, 20^{\circ}$ and $30^{\circ}$ to better visualize the surface topology.

Water Contact Angle Measurements: Water contact angle measurements were taken using a Ramé-Hart instrument, model \#590-F4. $10 \mu \mathrm{L}$ droplets of room temperature, deionized water were placed on the surface of the moth eye substrates. Measurements were taken in a 3 by 3 grid fashion, with 9 contact angle values recorded for each substrate (Figure SI 1). The best value and an average of all 9 values were determined for each substrate. The area of the $10 \mu \mathrm{L}$ droplets of water that were in contact with the moth eye surfaces were determined by the Cassie-Baxter equation using the average water contact angle values for each substrate. Advancing $\left(\mathrm{CA}_{\mathrm{Adv}}\right)$ and receding $\left(\mathrm{CA}_{\mathrm{Rec}}\right)$ water contact angles were determined using the "sessile drop" method.[15]

Seawater Incubation Tests: Artificial seawater (meeting ASTM standard D1141-98) was purchased from Lake Products Company, LLC, and used as received. The surface modified fused silica moth eye substrate was subjected to $20 \mathrm{hr}$ incubation in artificial seawater. Following incubation in the seawater, CA measurements were obtained in the same fashion as before (using deionized water, not seawater).

\section{Results and Discussion}

Surface Features and Characteristics. The nanopatterning of the fused silica substrates extended $\sim 800 \mathrm{~nm}$ into the surface of the material (Figure 1). The overall surface topology of the nanopatterned surface included randomly distributed, jagged and pointed features of various heights that protruded vertically from the surface of the fused-silica. The tips of the pointed features were tens of nanometers in diameter. These features were unaltered following the chemical surface modification process. Although the moth eye features were jagged and pointed, the PFOTS chemical modification prevented water droplets from being penetrated by these protrusions, and thus the modified fused silica moth eye surface is best described by the Cassie-Baxter surface wetting model rather than the Wenzel surface wetting model.[16, 17] 


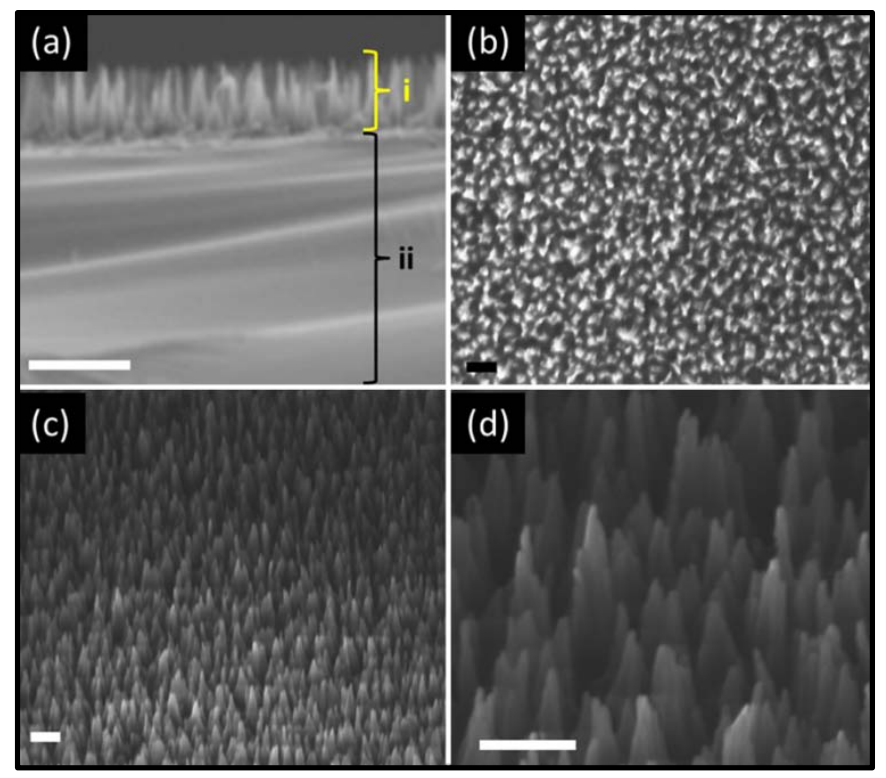

Figure 1. SEM images of the fused silica moth eye nanostructure surface features. (a) edge-on image showing the patterned surface (i) and the underlying fused silica substrate (ii). (b) top-down image, (c) \& (d) $30^{\circ}$ tilted angle images. Scale bars: (a) $=1$ $\mu \mathrm{m} ;$ (b), (c), (d) $=300 \mathrm{~nm}$.

Chemical Modification. In order to obtain optimal hydrophobicity, it is important to make the already hydrophilic surface optimally hydrophilic prior to chemical modification. It is equally important to use a cleaning process that does not damage the nanoscale features of the moth eye surface. To accomplish this cleaning task, the surfaces were cleaned using a plasma etching device under an $\mathrm{O}_{2(\mathrm{~g})}$ atmosphere. Following the plasma etch, the substrates were wafted for 30 seconds in a solution of hexanes that contained $0.5 \%$ PFOTS. This short wafting time in the hexanes/PFOTS solution prevented the buildup of non-specifically bound PFOTS on the surface of the material that could potentially cloud the surface, which would cause a decrease in transmission. Finally, the substrates were thermally cured at $120{ }^{\circ} \mathrm{C}$ to solidify bonding between the fused silica and the PFOTS molecules.

Superhydrophilicity was obtained by forming hydroxyl moieties on the fused silica surfaces via the plasma etch process. Because trichlorosilanes readily form self-assembled monolayers (SAMs) on hydroxyl containing surfaces, the etching process made the surface features more amenable to attachment with the trichlorosilane end of the PFOTS molecule.[12] As a consequence, this aligning of the PFOTS molecule on the surface caused the formation of an array of fluorine molecules (which are on the opposite end of the PFOTS molecule) to interface with the air. These fluorine molecules individually occupied a large spatial area, while their collective SAM alignment above the fused silica surface made the entire surface superhydrophobic. This superhydrophobic character can be seen as water droplets come in contact with the surface (SI Movie). 
Table 1. Water Contact Angle Data Before Chemical Modification

\begin{tabular}{|l|l|l|l|l|l|}
\hline Substrate & SA & CA & CA Adv $_{\text {Ad }}$ & CA $_{\text {Rec }}$ & H \\
\hline Fused Silica & $>10^{\circ}$ & $60.9 \pm 5.3^{\circ}$ & $54.4^{\circ}$ & $52.4^{\circ}$ & $2^{\circ}$ \\
\hline Fused Silica Moth & & & & & \\
eye & N/A & N/A & N/A & N/A & N/A \\
\hline
\end{tabular}

Water Contact Angle Analysis. Water contact angle (CA) measurements of cleaned fused silica and cleaned fused silica moth eye substrates were taken before and after the surface modification of each substrate. The nonmoth eye fused silica substrates returned CA values indicative of a hydrophilic surface (Table 1). For all moth eye substrates, the CA prior to surface modification was found to be nearly $0^{\circ}$ (i.e. superhydrophilic) because the fluid readily spread out along the substrate surface upon contact, rendering both the slide angle (SA) and CA immeasurable (Table 1).

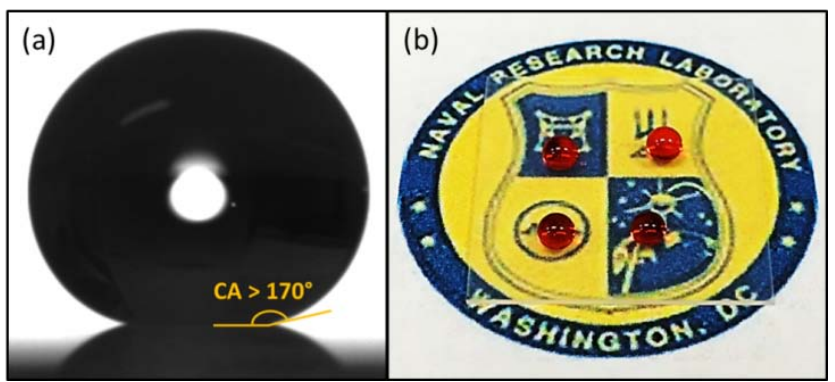

Figure 2. Images demonstrating the superhydrophobicity of the PFOTS treated moth eye surface. (a) representative photograph of the surface water contact angle, (b) red-dyed water droplets situated on the fused silica substrate, demonstrating the transparency of the substrate.

Following the surface modification, the fused silica moth eye surface exhibited superhydrophobicity, with the greatest $\mathrm{CA}$ being $176^{\circ}$ (Figure 2). The average $\mathrm{CA}_{\text {After }}$ was $172.8 \pm 4.5^{\circ}$, with the error being given in standard deviation of the data set. To determine the amount of water in contact with the low-wettability moth eye surface, the Cassie-Baxter equation was employed:

$$
\cos \mathrm{CA}_{\mathrm{avg}}=\varphi_{\mathrm{S}}\left(\cos \theta_{\mathrm{S}}+1\right)-1
$$

where $\mathrm{CA}_{\text {avg }}$ is the average water contact angle, $\theta_{\mathrm{S}}$ is the intrinsic water contact angle for unmodified, non-moth eye fused silica, and $\varphi_{\mathrm{S}}$ is the area of the substrate surface in contact with the water. Using this equation, it was determined that the fraction of the water droplets in contact with the moth eye surface was 0.01 (Table 2). By comparison, the Cassie-Baxter value for non-moth eye fused silica was determined to be 0.47 , indicating that 
much more of a single $10 \mu \mathrm{L}$ droplet of water was in contact with the non-moth eye surface than the fused silica with a moth eye surface topography (Table 2).

Table 2. Water Contact Angle Data After Chemical Modification

\begin{tabular}{|l|l|l|l|l|l|l|}
\hline Substrate & SA & CA & CA $_{\text {Adv }}$ & $\mathbf{C A}_{\text {Rec }}$ & H & Cassie-Baxter \\
\hline Fused Silica & $>10^{\circ}$ & $107.5 \pm 3.2^{\circ}$ & $105.3^{\circ}$ & $85.4^{\circ}$ & $19.9^{\circ}$ & 0.47 \\
\hline Fused Silica Moth & & & & & & \\
eye & $<1^{\circ}$ & $172.8 \pm 4.5^{\circ}$ & $164.4^{\circ}$ & $160.9^{\circ}$ & $3.5^{\circ}$ & 0.01 \\
\hline
\end{tabular}

The advancing and receding CAs for the moth eye substrate surface were determined to be $164.4^{\circ}$ and $160.9^{\circ}$, respectively. Using the equation

$$
\mathrm{H}=\mathrm{CA}_{\mathrm{Adv}}-\mathrm{CA}_{\mathrm{Rec}}
$$

in which $\mathrm{H}$ is the hysteresis value, $\mathrm{CA}_{\mathrm{Adv}}$ is the advancing contact angle, and $\mathrm{CA}_{\mathrm{Rec}}$ is the receding contact angle, $\mathrm{H}$ was determined to be $3.5^{\circ}$. This small $\mathrm{H}$ value suggested that the moth eye surface behaves as a near-ideal surface.

In addition to demonstrating superhydrophobicity following surface modification, the fused silica moth eye surface also maintained its transparency and anti-reflectance character (Figure 2b). The transmission and reflectance character of the chemically modified moth eye surface was compared to unmodified moth eye and non-moth eye fused silica substrates using UV-vis-NIR transmission spectroscopy (Figure 3).

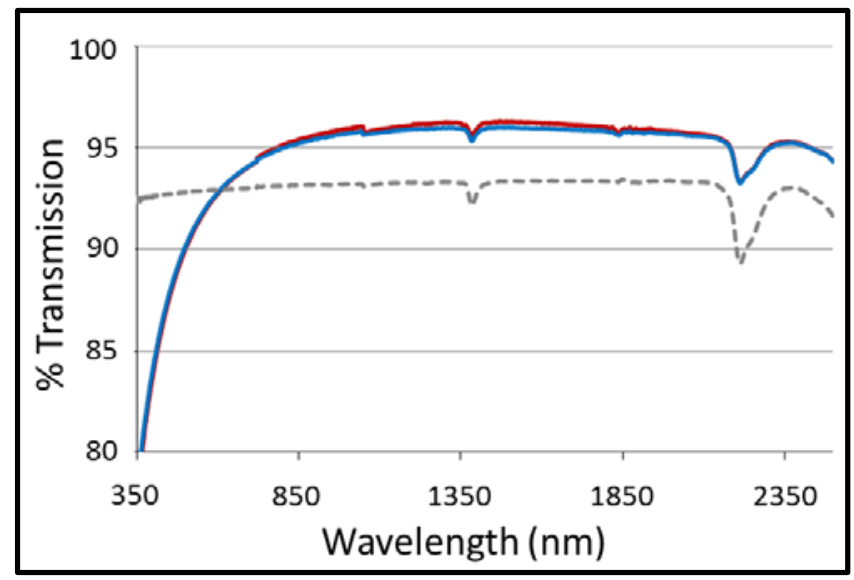

Figure 3. Transmission profile of fused silica moth eye without surface modification (red plot) and with surface modification (blue plot) compared to unmodified UV grade fused silica (gray, dashed plot). 
Transmission and Reflectance Properties. With transmittance of approximately $92 \%$ from the UV through the NIR (with the exception of the $-\mathrm{OH}$ overtone peaks seen at $\sim 1390$ and $\sim 2200 \mathrm{~nm}$ ), uncoated UV grade fused silica is a common standard for visible and infrared transmission quality. When fused silica is nanopatterned on one side, its transmittance can improve by as much as $4 \%$ for most of this range (Figure 3 ). As is typical for this type of AR structure, transmittance decreases in the UV as a result of scattering due to ARSS features size. Following the processing of the substrate, there was less than a $1 \%$ decrease in transmission for the majority of the region interrogated. This demonstrates that the fused silica moth eye substrates can undergo the chemical modification while retaining the desirable high transmission properties in the visible-NIR region of the spectrum (Figure 3).

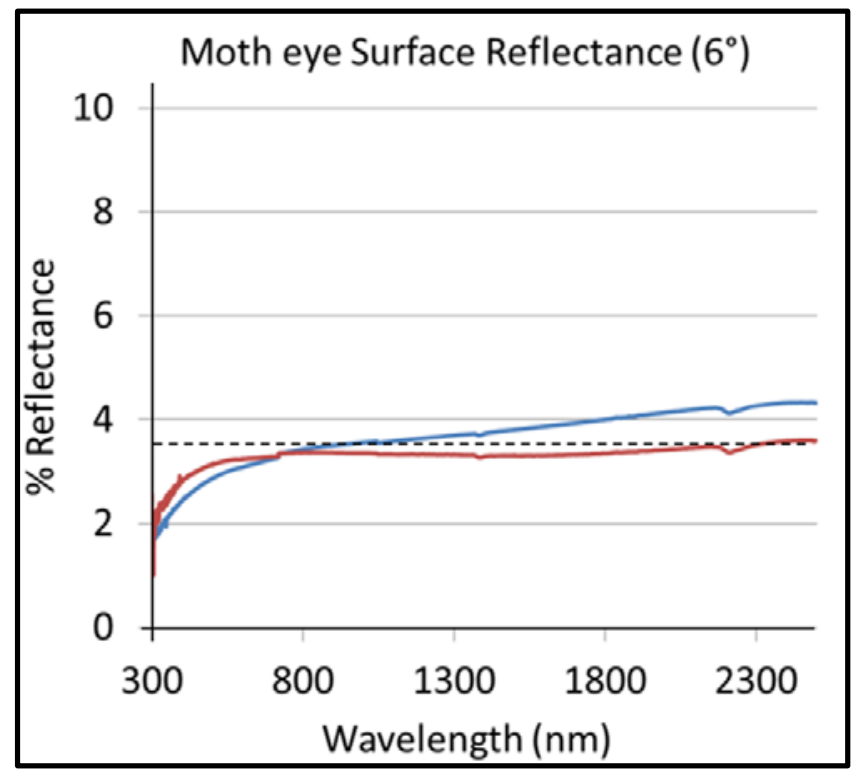

Figure 4. Representative reflectance data of fused silica moth eye before (red plot) and after (blue plot) surface modification at $6^{\circ}$. Horizontal dotted line represents the theoretical total reflectance of one-sided AR fused silica.

In addition to the IR transmission properties of fused silica moth eye, maintaining the reflectance properties of the material was also desirable. Data were taken of the moth eye substrate before and after the surface modification. As with the transmission properties, there was only a slight change in the reflectance of the moth eye substrates following chemical modification (Figure 4). The reflectance values obtained were comparable to the theoretical value of $3.5 \%$ for an ideal one-sided, UV grade, AR modified fused silica substrate. Ultimately, these data confirm that the moth eye nanopatterned surface topology improved the reflectance characteristics of the fused silica material, and this reflectance character is essentially maintained after chemical modification.

Seawater Submersion Tests. In order to evaluate the efficacy of the chemical modification in harsher conditions, clean fused silica substrates (both with and without moth eye) were immersed in artificial seawater for 
a period of $20 \mathrm{hrs}$, and CA values subsequently obtained for each substrate using deionized water. The submersion was performed both before and after chemical modification.

Table 3. Water Contact Angle Data After Seawater Immersion, But Before Chemical Modification

\begin{tabular}{|l|l|l|}
\hline Substrate & CA $_{\text {Sea }}{ }^{\dagger}$ & CA $_{\text {Son }}{ }^{\dagger}$ \\
\hline Fused Silica & $45.7 \pm 2.2^{\circ}$ & $58.4 \pm 3.2^{\circ}$ \\
\hline Fused Silica Moth eye & N/A & N/A \\
\hline
\end{tabular}

${ }^{\dagger} \mathrm{CA}_{\text {Sea }}$ is the contact angle after seawater immersion and $\mathrm{CA}_{\text {Son }}$ is the contact angle after sonication.

Prior to chemical modification, incubating the non-moth eye fused silica in seawater yielded contact angles $\left(\mathrm{CA}_{\text {Sea }}\right.$ ) of $\sim 45^{\circ}$ (Table 3), which was much less than the CA value of $\sim 60^{\circ}$ determined for a clean sample (Table 1). However, the native surface hydrophilicity could be recovered upon 5 minute sonication $\left(\mathrm{CA}_{\mathrm{Son}}\right)$ in deionized water (Table 3). Interestingly, incubating the fused silica moth eye in seawater still yielded an extremely hydrophilic surface. The water contact angle following seawater incubation $\mathrm{CA}_{\text {Sea }}$ was still less than $1^{\circ}$ and essentially immeasurable (Table 3).

Table 4. Water Contact Angle Data After Seawater Immersion \& After Chemical Modification

\begin{tabular}{|l|l|l|}
\hline Substrate & $\mathbf{C A}_{\text {Sea }}$ & CA $_{\text {Son }}$ \\
\hline Fused Silica & $104.2+5.1^{\circ}$ & $101.4 \pm 8.4^{\circ}$ \\
\hline Fused Silica Moth eye & $157.1 \pm 9.4^{\circ}$ & $166.4 \pm 5.7^{\circ}$ \\
\hline
\end{tabular}

Newly cleaned and chemically modified fused silica substrates were incubated in seawater for 20 hours. For the non-moth eye fused silica, all values, whether obtained after surface modification (Table 2) or obtained before/after seawater incubation (Table 4), were within error of each other. This was likely due to the fact that there were no nanoscale features on the surface to alter the surface response to chemical modification. 


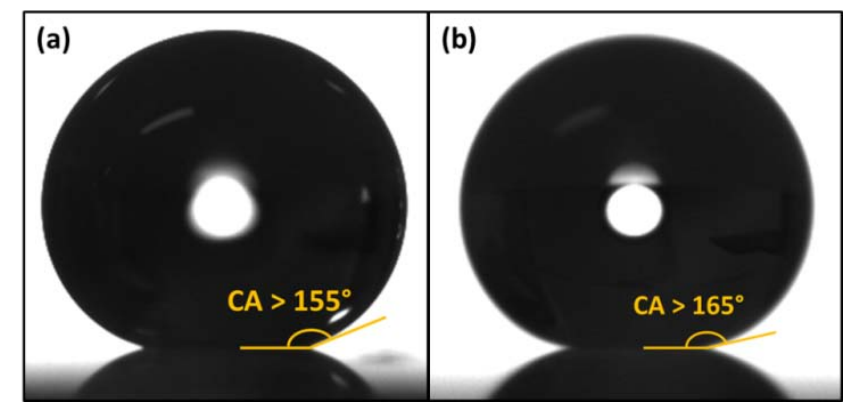

Figure 5. Images demonstrating the superhydrophobicity of the moth eye surface (a) after incubation in seawater, and (b) following sonication to remove the seawater salts.

The seawater incubation had a noticeable impact on the CA of the chemically modified fused silica moth eye, however the surface remained superhydrophobic (Figure 5), with an average $\mathrm{CA}_{\text {Sea }}$ of $157.1 \pm 9.4^{\circ}$ (Table 4). The CA value was improved following 5 minute sonication in deionized water, with an average $\mathrm{CA}_{\text {Son }}$ value of $166.4 \pm 5.7^{\circ}$ and a best value of $173.0^{\circ}$ (Figure $5 \mathrm{~b}$ ). These results indicated that the chemical modification of the fused silica moth eye substrate was quite stable.

\section{Conclusions}

The work presented demonstrates fused silica can be patterned with randomly distributed nanostructures to impart anti-reflection characteristics, while maintaining the desirable infrared transmission properties of fused silica. Furthermore, these structures can be chemically modified in such a manner as to decrease the surface energy and to impart superhydrophobic character to the surface. Finally, the chemically modified, randomly patterned moth eye surface also appeared to be effective in either repelling or masking the infiltration of seawater salt components. Overall, our process is straightforward, scalable and applicable in various commercial and military applications such as self-cleaning materials (e.g. materials with high contact angle, superhydrophobic surfaces that cause the beading and rolling of water droplets at shallow slide angles), and applications involving high transmission optical coatings. Furthermore, the methodology outlined could be readily applied to other substrate materials, also rendering them superhydrophobic while having minimal adverse effects on their transmission and reflectance properties.

\section{ACKNOWLEDGMENTS}

We would like to kindly thank Dr. Erick Iezzi for the use of his labs' goniometer. The work was supported by the Jerome \& Isabella Karle Fellowship. The views are those of the authors and do not represent the opinion or policy of the US Navy or Department of Defense. 


\section{AUTHOR INFORMATION}

\section{Corresponding Author}

*E-mail: darryl.boyd@nrl.navy.mil

\section{Author Contributions}

The manuscript was written through contributions of all authors. All authors have given approval to the final version of the manuscript.

\section{REFERENCES}

[1] C.G. Bernhard, Endeavour, 26 (1967), pp. 79-84.

[2] D.S. Hobbs, B.D. MacLeod, Design, fabrication, and measured performance of anti-reflecting surface textures in infrared transmitting materials, 2005, pp. 349-364.

[3] H.K. Raut, S.S. Dinachali, Y.C. Loke, R. Ganesan, K.K. Ansah-Antwi, A. Gora, E.H. Khoo, V.A. Ganesh, M.S. Saifullah, S. Ramakrishna, ACS nano, 9 (2015), pp. 1305-1314.

[4] F. Galeotti, F. Trespidi, G. Timo, M. Pasini, ACS applied materials \& interfaces, 6 (2014), pp. 5827-5834.

[5] K. Nakata, M. Sakai, T. Ochiai, T. Murakami, K. Takagi, A. Fujishima, Langmuir : the ACS journal of surfaces and colloids, 27 (2011), pp. 3275-3278.

[6] X. Qian, N. Wang, Y. Li, J. Zhang, Z. Xu, Y. Long, Langmuir : the ACS journal of surfaces and colloids, 30 (2014), pp. $10766-10771$.

[7] S. Ji, K. Song, T.B. Nguyen, N. Kim, H. Lim, ACS applied materials \& interfaces, 5 (2013), pp. 10731-10737.

[8] C.M. Florea, L.E. Busse, S.S. Bayya, B. Shaw, I.D. Aggarwal, J.S. Sanghera, Recent advancements in anti-reflective surface structures (ARSS) for near- to mid-infrared optics, 2013, pp. 87080P-87080P-87017.

[9] L.E. Busse, C.M. Florea, J.A. Frantz, L.B. Shaw, I.D. Aggarwal, M.K. Poutous, R. Joshi, J.S. Sanghera, Opt. Mater. Express, 4 (2014), pp. 2504-2515.

[10] J. Sanghera, C. Florea, L. Busse, B. Shaw, F. Miklos, I. Aggarwal, Optics express, 18 (2010), pp. 26760-26768.

[11] K.C. Park, H.J. Choi, C.H. Chang, R.E. Cohen, G.H. McKinley, G. Barbastathis, ACS nano, 6 (2012), pp. $3789-3799$.

[12] M. Psarski, J. Marczak, G. Celichowski, G. Sobieraj, K. Gumowski, F. Zhou, W. Liu, centr.eur.j.phys., 10 (2012), pp. $1197-1201$.

[13] T. Aytug, A.R. Lupini, G.E. Jellison, P.C. Joshi, I.H. Ivanov, T. Liu, P. Wang, R. Menon, R.M. Trejo, E. Lara-Curzio, S.R. Hunter, J.T. Simpson, M.P. Paranthaman, D.K. Christen, Journal of Materials Chemistry C, 3 (2015), pp. 5440-5449.

[14] A. Tolga, T.S. John, R.L. Andrew, M.T. Rosa, E.J. Gerald, N.I. Ilia, J.P. Stephen, A.H. Daniel, O.W. Kyle, K.C. David, R.H. Scott, J.A. Haynes, Nanotechnology, 24 (2013), p. 315602.

[15] J.T. Korhonen, T. Huhtamaki, O. Ikkala, R.H. Ras, Langmuir : the ACS journal of surfaces and colloids, 29 (2013), pp. $3858-3863$.

[16] A.B.D. Cassie, S. Baxter, Transactions of the Faraday Society, 40 (1944), pp. 546-551.

[17] A. Marmur, Langmuir : the ACS journal of surfaces and colloids, 19 (2003), pp. 8343-8348. 
Modification of Nanostructured Substrates For Use as Superhydrophobic, IR-Transmissive, AntiReflective Surfaces

Darryl A. Boyd, Jesse A. Frantz, Shyam S. Bayya, Lynda E. Busse, Woohong Kim, Ishwar Aggarwal, Menelaos Poutous, and Jasbinder S. Sanghera

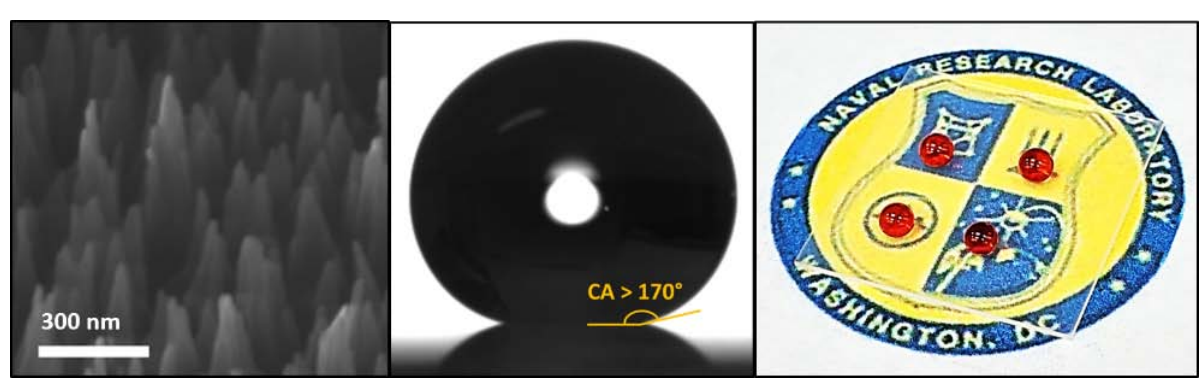




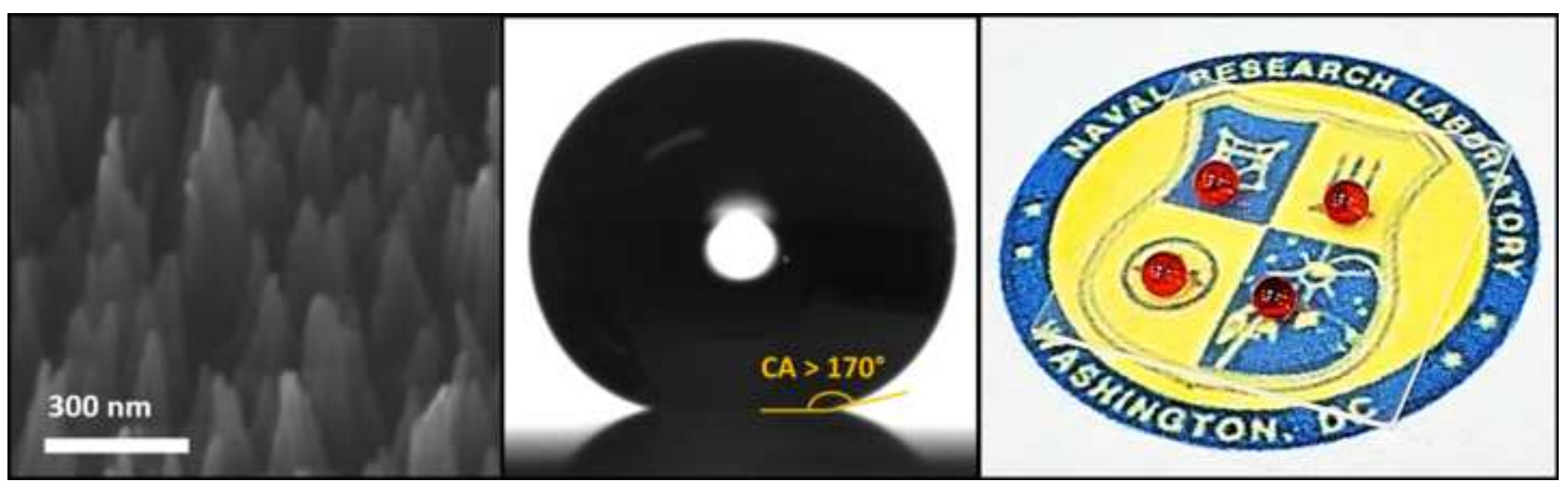

